\title{
Spatial distribution of monogenean gill parasites of Parachanna obscura (Günther, 1861) - Channidae - in Lake Ossa (Edéa, Cameroon)
}

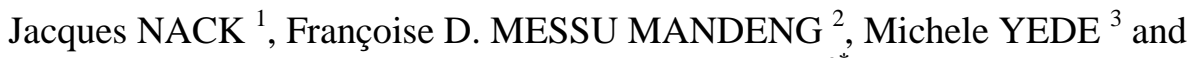 \\ Charles F. BILONG BILONG ${ }^{3^{*}}$ \\ ${ }^{1}$ University of Douala, Institute of Fisheries and Aquatic Sciences, P.O. Box 7236 Douala, Cameroon. \\ ${ }^{2}$ University of Yaoundé I, Higher Teacher Training College, P.O. Box 47 Yaoundé, Cameroon. \\ ${ }^{3}$ University of Yaoundé I, Faculty of Science P.O. Box 812 Yaoundé, Cameroon. \\ *Corresponding author; E-mail: admiyede@yahoo.fr; bilong_bilong@yahoo.com
}

\begin{abstract}
Parasites are a danger for fish even more in natural environment than in farming conditions. Knowledge of their biology of is important for prevention. The objective of this study was to investigate the distribution of monogenean gill community of Parachanna obscura (Pisces, Channidae). Monogenean species were identified using the morphology of haptoral and genital sclerotized parts. Appropriate statistical tests were used to compare prevalence and intensity of parasites. In Lake Ossa (south Cameroon), $P$. obscura individuals were in relatively good condition $(\mathrm{K}=1,4 \pm 0,15)$ although they were infected by five monogenean species (Eutrianchoratus magnus, E. minutus, E. imbachi, E. chibami and E. malleus). Male and female fish harbor similar parasite intensity. There was not statistical difference in the infection patterns of different host sizes, although big or old fish seemed to accumulate parasites over time. The distribution of helminthes on the gills did not revealed host side preference. There is no evidence of competition between species of Eutrianchoratus in their distribution areas, but the filaments of different gill arches exert a progressive (antero-posterior) sifting effect on the infecting larvae. This study proves once again that in natural environment parasite intensities are very low or low and this state minimizes competition between monogeneans species.
\end{abstract}

(C) 2018 International Formulae Group. All rights reserved.

Keywords: Ancyrocephalidae, Eutrianchoratus, sifting effect, prevalence, intensity, fish.

\section{INTRODUCTION}

Channidae, formerly Ophiocephalidae, are cylindrical perciform fishes found in Africa and Asia. Their accessory breathing organ, in the form of two pharyngeal suprabranchial chambers, enables them to utilize atmospheric air and survive out of water for considerable periods. A single genus, Parachanna (Teugels and Daget, 1984) is present in Africa with three species represented in Lower Guinea (Teugels, 2007), among which two, $P$. africana (Steindadener, 1879) and P. obscura (Gunther, 1961), are found in Cameroon. Parachanna obscura, also named "snakehead", is a synonym of Channa obscura, C. obscurus, Paraophiocephalus obscurus, Paraphicephalus obscurus and Ophiocephalus obscurus. It is distributed from the Zaire basin through West Africa, as from the Senegal River in the West 
to the Nile in the East (Stiassny et al., 2007; Teugels, 2007). It is a piscivorous/predatory fish which lives in the bank vegetation of the main river channel or in the vegetated (swampy) areas in the flood plain, pools and lagoons (Olasunkanmi and Ipinmoroti, 2014). It has been used or studied for use in African aquaculture (Odo et al., 2011) like others african native fish (Zango et al., 2016; Mukabo Okito et al., 2017) and to regulate cichlid populations (Kpogue et al., 2013). This robust and tolerant species reproduces throughout the year with a peak during or just after rainfall (Whenu and Fagade, 2012; Kpogue et al., 2013). It reachs a maximum size of $455 \mathrm{~mm}$ total length (Teugels, 2007; Vodounnou et al., 2017) and therefore represents a good source of survival proteins; moreover its delicious flesh is appreciated by local populations.

The gill filaments of P. obscura in the Nyong basin (Cameroon) are parasitized by five monogenean species (Bilong Bilong, 1995): Eutrianchoratus magnus (Paperna, 1969); E. minutus (Paperna, 1969); E. imbachi (Dossou and Euzet, 1984); E. chibami (Bilong Bilong, et al., 1994); and E. malleus (Bilong Bilong et al., 1994). The ecological aspects of the parasitism of this host by these ancyrocephalids remain unstudied. In fact, fish parasites form interesting and little-used subjects for studies on communities and their ecology (Lekeufack Folefack and Fomena, 2013; Tombi et Bilong Bilong, 2013). The main objectives of this study was to provide informations on some ecological aspects of the monogenean gill community of Parachanna obscura by investigating how these parasitic helminthes exploit their hosts.

\section{MATERIALS AND METHODS Study area}

Specimens of $P$. obscura were caught using gill nets from July to November 2014 during the rainy season in Lake Ossa

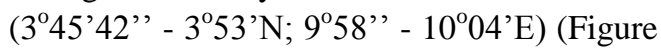
1), located $20 \mathrm{~km}$ to the west of the city of Edéa and $30 \mathrm{~km}$ from the Atlantic Ocean (Bahanak et al., 2016).

\section{Sampling methods}

Fish captured were immediately immerged in a $10 \%$ formalin solution to fix monogeneans in situ and prevent their loss due to abundant host post-mortem excretion of mucus (Bilong Bilong, 1995). After few minutes, a $1 \mathrm{~cm}$ abdominal buttonhole (incision) was realized on each specimen to later identify its sex. Fishes were then transported to the laboratory for parasite and host investigations.

\section{Methods and data collection tools}

In the laboratory, the standard length of each fish specimen was measured in millimeters (mm); the latter was then immerged in tap water to reduce formalin contamination. Gill arches from both the right and left sides, numbered I to IV in the anteroposterior direction, were removed separately and placed in different labeled Petri dishes containing tap water.

Monogeneans were dislodged from the gill filaments with a needle under stereoscopic microscope. They were stained in-between slides and cover slips into a drop of haematoxylin eosin (Bilong Bilong, 1986), then identified at 40x magnification under a microscope after Paperna (1969), Dossou and Euzet (1984) and Bilong Bilong et al. (1994) by the morphology and size of sclerotized pieces of the haptor and copulatory organs. In this transverse analysis, the terms prevalence, intensity (I), mean intensity (MI) and abundance are defined after Bush et al. (1997), infracommunity and xenocommunity after Combes (1995), transverse gradient after Rohde (1977), core and satellite species after Kooskivaara and Valtonen (1991), common, intermediate and rare species after Valtonen et al. (1997). The mean intensity (even the intensity) is categorized in our environment as follows: very low $(\mathrm{MI} \leq 10)$, low $(10<\mathrm{MI} \leq$ $50)$, average $(50<\mathrm{MI} \leq 100)$ and high $(\mathrm{MI}>$ 100) after Bilong Bilong and Njiné (1998). Parasitic individuals typically exhibit an aggregated distribution among available host specimens such that most of the latter harbor no or few parasites and few hosts harbor many parasites (Rõzsa et al., 2000); therefore the 
prevalence and mean intensity are respectively given with confidence interval or bootstrap confidence limits to $95 \%$ probability.

\section{Data analysis}

The index of discrepancy D quantifies the difference between an observed parasite distribution and a hypothetical distribution that corresponds to the ideal case where all hosts harbor the same number of parasites. It measure the concept of aggregation as intuitively perceived by most parasitologists (Poulin, 1998). The prevalences were compared using Fisher's exact test and mean intensities by a bootstrap test or by Mood's medium test. APS statistical comparisons were realized using the QP30 software (Rõzsa et al., 2000; Reiczigel et al., 2005). The parasites overlap at the level of the gill arch, sector and zone (Figure 2) was estimated using the Forbes index followed by Khi-2 test. The Forbes index measures the deviation of an association at random: $F=1$ means that parasites are randomly associated; $\mathrm{f}=2$ means that the association of the species is twice more favorable than fortuity; $\mathrm{F}<1$ means that the parasites association is less than predicted chance. Differences were considered as significant when $\mathrm{P}<0.05$. Analyses concerned only core and secondary species. Since in a community satellite or rare species are little or no structuring (Holmes, 1987; Combes, 1995).

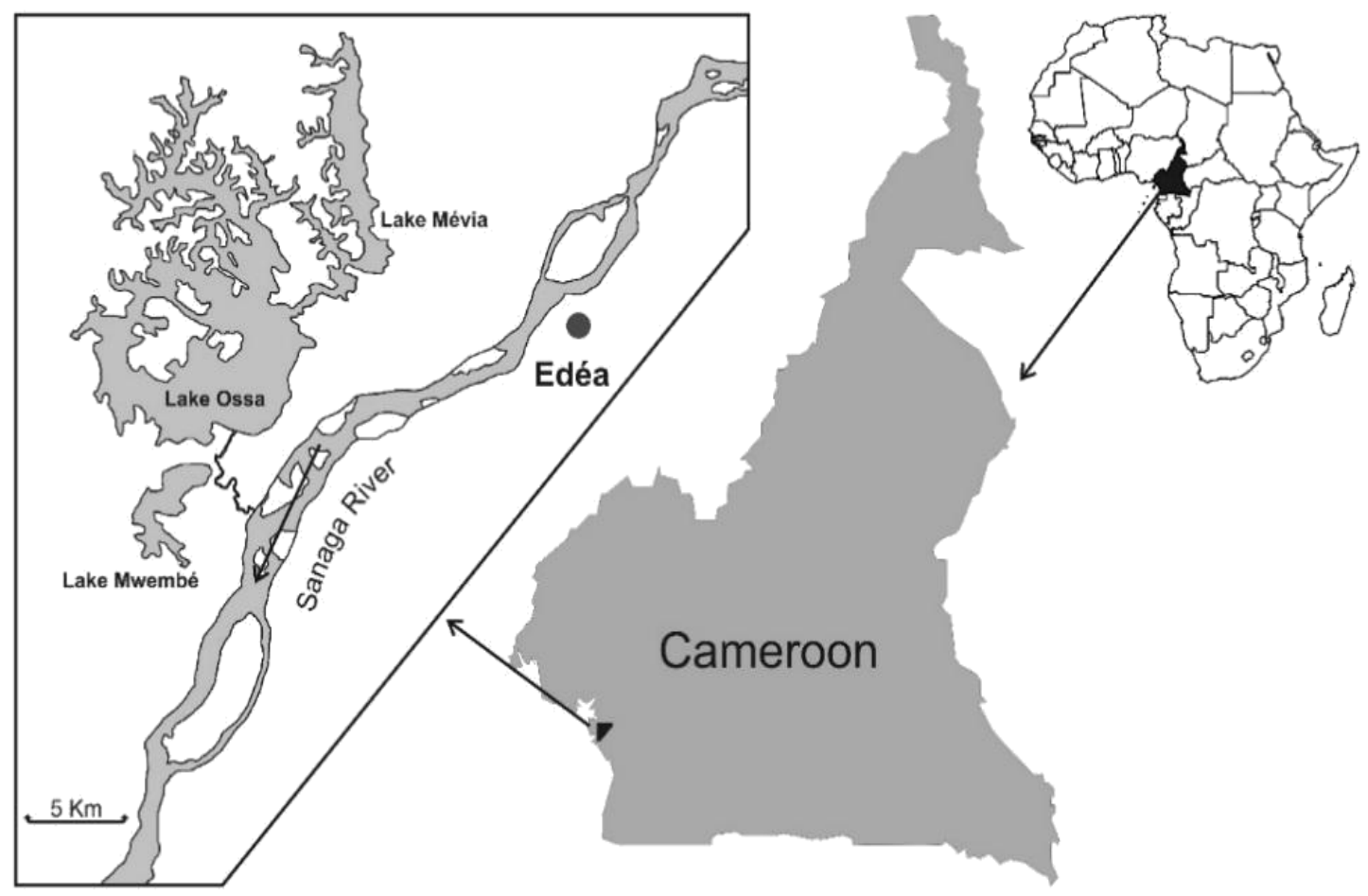

Figure1: Geographic localization of Lake Ossa (After Bahanak et al., 2016 modified). 


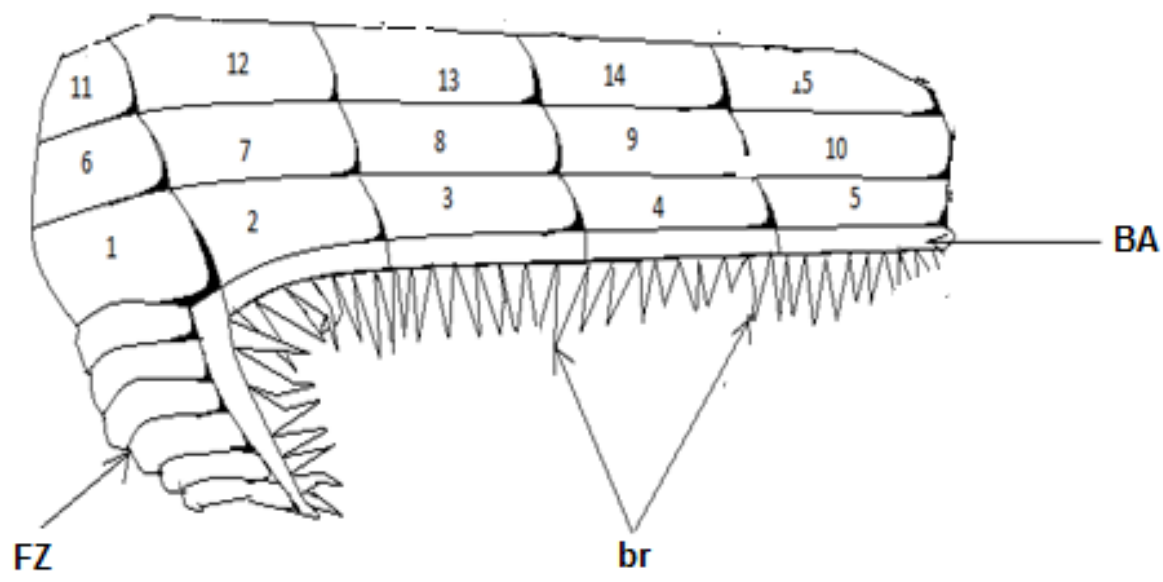

Figure 2: Sectoral and zonal delimitation of gill areas of Parachanna obscura.

Legend: 1 to $15=$ stations; 1,6 , and $11=$ stations of the dorsal sector; 2,7 and $12=$ stations of the medio-dorsal sector; 3,8 and $13=$ stations of the median sector; 4,9 and $14=$ stations of the medioventral sector; 5, 10 and 15 stations of the ventral sector; 1,2,3,4 and 5: basal zone; 6,7,8,9 and 10 median zone; $11,12,13,14$ and 15 distal zone; $\mathrm{BA}=$ bone arch; $\mathrm{br}=$ branchiospines; $\mathrm{ZF}=$ fibrous zone.

\section{RESULTS}

A total of 50 specimens (30 females, 20 males: sex-ratio $=1.5$ ) of Parachanna obscura were examined. Their standard length ranged from 200 to $300 \mathrm{~mm}$. Fish were generally in good physiological condition since their condition factor $\mathrm{K}$ was averagely $1.40(1.17$ - 1.71) \pm 0.15 . At least 9847 monogeneans were collected from this fish sample: 4694 Eutrianchoratus minutus (47.7\%), 3278 E. imbachi (33.3\%), 1610 E. chibami (16.3\%), 246 E. malleus (2.5\%) and 19 E. magnus (0.2\%). The prevalence (prev.), mean intensity and discrepancy index (D) of these parasite species are summarized in Table 1. No difference was found in the infection between male and female hosts, either for prevalences or mean intensities (Table 2). At the xenocommunity level, only one fish individual was found free of any parasite species $($ Prev. $=98.00 \%(89.35$ 99.89), the mean number (MN) of helminthes per infected host $=100.65 ; \mathrm{D}=0.462)$. The highest number of monogeneans harbored by a specimen of $P$. obscura was 403 . As a function of the length classes, $\mathrm{MN}=77.61$ (54.43 - 122.96) for the host length class [200 - 250[, MN = $123.81(88.33-172.38)$ for host length class $[250-300$ [and $\mathrm{MN}=109.60$ (44.80 - 221.80) for host length class [300 350[. These mean values were not significantly different $(P=0.301)$, even if hosts of the length class [250 - 300[ averagely seemed to accumulate more helminth individuals. This was the pattern for $E$. chibami and E. imbachi, while for E. minitus the mean parasitic load significantly $(\mathrm{P}=$ 0.001) increased up to the highest level in the host length class [300 - 350[(Table 3). No side effect was noticed in the infection of $P$. obscura either for the rate of parasitism or the mean number of parasites per infected side (Table 4). Based on this result, the study of monogenean distribution in the transverse gradient had therefore considered indifferently only one side per fish.

\section{Distribution at the level of arches}

The Mood's median test comparing median intensities revealed statistical differences between gill arches only for $E$. chibami $(\mathrm{P}=0.000)$, E. imbachi $(\mathrm{P}=0.004)$ and E. minitus $(\mathrm{P}=0.003)$ (Table 5). Therefore the comparison continued only with these three species. Eutrianchoratus minitus and E. 
imbachi concentrated antero-posteriorly on gill arches with the following pattern

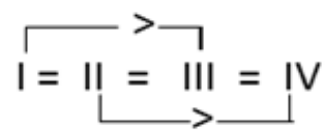

As for E. chibami, although there was a significant difference between the four gill arches, the multiple comparisons did not show any difference, its distribution pattern was $\mathrm{I}=$ $\mathrm{II}=\mathrm{III}=\mathrm{IV}($ Table 6$)$.

\section{Distribution at the hemibranches level}

For E. minitus, the anterior hemibranches were statistically equally colonized (prevalence). But, anterior hemibranches were more exploited than the posterior ones (Table 7a). The pattern of gill occupation of this monogenean species was:

$$
\left\|_{A}=I_{A}=\right\| I_{A}=I V_{A}>I_{P}=I I_{P}=\|_{P}=I V_{P}
$$

Similarly, the abundance of this helminth species didn't significantly vary between anterior hemibranch in the one hand, and posterior hemibranch in the other hand (Table 7b). But, the parasitism decreases slightly in the antero-posterior direction as the following model:

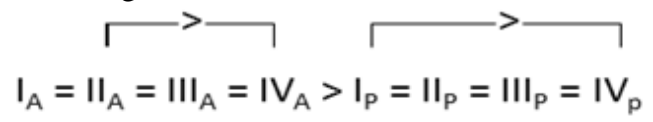

For E. imbachi, the prevalence analysis (Table 7c) also showed that the anterior hemibranches on one hand and the posterior hemibranches on the other hand were statistically similarly colonized. However, the anterior hemibranches are more occupied than the posterior ones. The pattern of occupation was:

$$
\mathrm{II}_{A}=\mathrm{I}_{A}=\mathrm{III} \mathrm{I}_{\mathrm{A}}=\mathrm{IV} \mathrm{A}_{\mathrm{A}}>\mathrm{I}_{\mathrm{P}}=\mathrm{III} \mathrm{I}_{\mathrm{P}}=\mathrm{II}_{\mathrm{P}}=\mathrm{IV} \mathrm{V}_{\mathrm{P}}
$$

The study of abundance of this monogenean species also revealed the anteroposterior model of colonization and, the posterior hemibranches harbored less parasites (Table 7d). The model of the infection was:

$$
\begin{aligned}
& \longrightarrow \longrightarrow \longrightarrow
\end{aligned}
$$

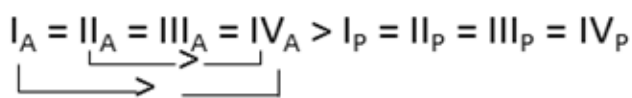

This was the same for E. chibami. The model of infection according with the prevalence (Table 7e) was:

$$
\mathrm{I}_{\mathrm{A}}>\mathrm{II}_{\mathrm{A}}=\mathrm{III}_{\mathrm{A}}=\mathrm{IV}_{\mathrm{A}}=\mathrm{I}_{\mathrm{P}}>\mathrm{II}_{\mathrm{P}}=\mathrm{III}_{\mathrm{P}}=\mathrm{IV}_{\mathrm{p}}
$$

While that according with the abundance was:

$I_{A}=I_{A}=I I_{A}=I V_{A}=I_{P}>I_{P}=I I_{P}=I V_{P}$

The posterior hemibranches of arch I harbored the same number of E. chibami individuals than the anterior hemibranch of arch IV (Table 7f).

At the sectorial level, for many parasitic pairs, the values of the Forbes Index were not very different from the unity. Therefore, these parasitic associations seem to occur randomly. Nevertheless, the cooccurrence of the pairs E. minitus / E. imbachi (sector 5: arches 3 and 4), E. minitus / E. chibami (sector 1: arch 4; sector 2: arch 4; sector 3: arch 2; sector 5: arches 2 and 3) and E. imbachi / E. chibami (sector 1, arch3; sector 2; arch 3; sector 4: $\operatorname{arch} 4$ ) was higher than predicted (Table 8).

At the zonal level, for the different pairs of parasitic species, the values of the Forbes index were close to 1 showing that in most cases the distribution of these parasites was done at random, except for pairs: $E$. minitus / E. imbachi (basal zone: arch 4), E. minitus / E. chibami (median zone: arc 3, basal zone: arc 4), and E. imbachi / E. chibami (distal zone: arc 1, 3 and 4) where positive associations seemed more frequent than predicted (Table 9). Eutrianchoratus minitus concentrated much more in the median zone of arches 1,2, 3 and less in arch 4 (basal and dorsal areas). 
Table 1: Prevalence and mean intensity of monogenean species of $P$. obscura.

\begin{tabular}{llll}
\hline Parasite species & Prevalence $(\mathbf{C I})$ & Mean Intensity (BC) & Discrepancy index (D) \\
\hline E. chibami & $90.0(78.18-96.68)$ & $17.89(11.11-35.60)$ & 0.623 \\
E. imbachi & $98.0(89.35-99.95)$ & $33.45(25.71-44.00)$ & 0.501 \\
E. magnus & $24.0(13.06-37.17)$ & $1.58(1.17-1.92)$ & 0.794 \\
E. malleus & $58.0(43.20-71.82)$ & $4.24(3.03-5.72)$ & 0.669 \\
E. minutus & $96.0(86.28-99.52)$ & $48.90(37.63-65.42)$ & 0.496 \\
\hline
\end{tabular}

CI: confidence interval; BC: bootstrap confidence limit.

Table 2: Prevalence and mean intensity of monogenean species as a function of $P$. obscura sex.

\begin{tabular}{|c|c|c|c|c|c|c|c|}
\hline \multirow{2}{*}{$\begin{array}{l}\text { Parasites } \\
\text { species }\end{array}$} & \multicolumn{2}{|c|}{ Prevalence } & \multicolumn{2}{|c|}{ Mean intensity } & \multirow[t]{2}{*}{ T value } & \multicolumn{2}{|c|}{ P value } \\
\hline & $\mathbf{M}$ & $\mathbf{F}$ & $\mathbf{M}$ & $\mathbf{F}$ & & $\begin{array}{c}\text { Fisher's } \\
\text { exact test }\end{array}$ & $\begin{array}{c}\text { Bootstrap } \\
\text { T Test }\end{array}$ \\
\hline E. chibami & 91.7 & 85.7 & 11.33 & 35.92 & 1.341 & 0.611 & 0.275 \\
\hline E. imbachi & 97.2 & 100 & 36.89 & 24.86 & 1.485 & 1.000 & 0.158 \\
\hline E. magnus & 27.8 & 14.3 & 1.50 & 2.00 & -2.236 & 0.468 & 0.124 \\
\hline E. malleus & 61.1 & 50.0 & 4.00 & 5.00 & -0.639 & 0.534 & 0.520 \\
\hline E. minutus & 92.9 & 97.2 & 51.35 & 42.31 & 0.623 & 0.486 & 0.558 \\
\hline
\end{tabular}

Table 3: Mean Intensity as a function of host size class.

\begin{tabular}{|c|c|c|c|}
\hline \multirow[t]{2}{*}{ Parasite species } & \multicolumn{3}{|c|}{ Host size (mm) } \\
\hline & {$[200-250]$} & {$[250-300]$} & {$[300-350]$} \\
\hline E. minutus & $\mathrm{MI}=34.50 ; \mathrm{n}=22$ & $\mathrm{MI}=59.9 ; \mathrm{n}=21$ & $\mathrm{MI}=69.0 ; \mathrm{n}=5$ \\
\hline E. imbachi & $\mathrm{MI}=33,15 ; \mathrm{n}=23$ & $\mathrm{MI}=34,29 ; \mathrm{n}=21$ & $\mathrm{MI}=31.40 ; \mathrm{n}=5$ \\
\hline E. chibami & $\mathrm{MI}=9.90 ; \mathrm{n}=21$ & $\mathrm{MI}=29.42 ; \mathrm{n}=19$ & $\mathrm{MI}=7.60 ; \mathrm{n}=5$ \\
\hline Infracommunity & $\mathrm{MN}=77.61 ; \mathrm{n}=23$ & $\mathrm{MN}=123.81 ; \mathrm{n}=21$ & $\mathrm{MN}=109.60 ; \mathrm{n}=5$ \\
\hline
\end{tabular}

MN: mean number; MI: mean intensity; n: number of fishes. 
Table 4: Prevalence and mean intensity of monogenean species as a function of host side

\begin{tabular}{lccccccc}
\hline $\begin{array}{l}\text { Parasites } \\
\text { species }\end{array}$ & \multicolumn{2}{c}{ Prevalence } & \multicolumn{2}{c}{ Mean intensity } & T value & \multicolumn{2}{c}{ P value } \\
\cline { 2 - 3 } & $\mathbf{R}$ & $\mathbf{L}$ & $\mathbf{R}$ & $\mathbf{L}$ & & $\begin{array}{l}\text { Fisher's } \\
\text { exact test }\end{array}$ & $\begin{array}{l}\text { Bootstrap T } \\
\text { test }\end{array}$ \\
\hline E. chibami & 80.0 & 88.0 & 10.70 & 8.57 & 0.497 & 0.414 & 0.646 \\
E. imbachi & 92.0 & 94.0 & 17.00 & 18.23 & -0.336 & 1.000 & 0.744 \\
E. magnus & 16.0 & 14.0 & 1.25 & 1.29 & -0.145 & 1.000 & 0.776 \\
E. malleus & 44.0 & 48.0 & 2.26 & 2.96 & -0.983 & 0.841 & 0.343 \\
E. minutus & 92.0 & 94.0 & 25.52 & 24.96 & 0.107 & 1.000 & 0.909 \\
\hline
\end{tabular}

$\mathrm{R}=$ right side, $\mathrm{L}=$ left side.

Table 5: Infection rate and mean parasites load of P. obscura per arch.

\begin{tabular}{|c|c|c|c|c|c|c|}
\hline \multirow{3}{*}{ Parasite species } & \multirow[t]{3}{*}{ Arch } & \multirow{2}{*}{$\begin{array}{l}\text { Infection } \\
\text { rate }(\%) \\
\end{array}$} & \multicolumn{2}{|c|}{ Parasite } & \multicolumn{2}{|r|}{ P-value } \\
\hline & & & \multirow{2}{*}{$\begin{array}{c}\text { Mean } \\
\text { number }\end{array}$} & \multirow[t]{2}{*}{ Medium } & \multirow[t]{2}{*}{ Fisher } & Mood's \\
\hline & & & & & & médian test \\
\hline & I & 80.0 & 9.95 & 5.0 & & \\
\hline & II & 76.0 & 5.39 & 2.5 & 0.036 & 0.000 \\
\hline \multirow[t]{5}{*}{ E. chibami } & & 58.0 & 3.59 & 2.0 & & \\
\hline & III & & & & & \\
\hline & IV & 60.0 & 3.27 & 2.0 & & \\
\hline & I & 88.0 & 13.82 & 10.5 & & \\
\hline & II & 86.0 & 10.33 & 7.0 & 0.658 & 0.004 \\
\hline \multirow[t]{5}{*}{ E. imbachi } & & 88.0 & 7.86 & 5.5 & & \\
\hline & III & & & & & \\
\hline & IV & 80.0 & 6.03 & 4.0 & & \\
\hline & I & 10.0 & 1.2 & 1.0 & & \\
\hline & II & 6.0 & 1.0 & 1.0 & 0.702 & 0.515 \\
\hline \multirow[t]{5}{*}{ E. magnus } & & 12.0 & 1.0 & 1.0 & & \\
\hline & III & & & & & \\
\hline & IV & 6.0 & 1.33 & 1.0 & & \\
\hline & I & 36.0 & 1.94 & 1.5 & & \\
\hline & II & 38.0 & 1.74 & 1.0 & 0.744 & 0.393 \\
\hline \multirow[t]{5}{*}{ E. malleus } & & 34.0 & 2.18 & 2.0 & & \\
\hline & III & & & & & \\
\hline & IV & 28.0 & 1.29 & 1.0 & & \\
\hline & I & 90.0 & 20.38 & 13.0 & & \\
\hline & II & 92.0 & 15.61 & 10.0 & 0.692 & 0.003 \\
\hline \multirow[t]{3}{*}{ E. minutus } & & 88.0 & 9.27 & 6.5 & & \\
\hline & III & & & & & \\
\hline & IV & 84.0 & 7.24 & 6.0 & & \\
\hline
\end{tabular}


Table 6: Multiple comparison of mean parasite loads between arches.

\begin{tabular}{|c|c|c|c|}
\hline Arch II & $\begin{array}{l}1.184 \\
0.254\end{array}$ & & \\
\hline Arch III & $\begin{array}{l}3.383 \\
0.003^{*}\end{array}$ & $\begin{array}{l}2.113 \\
0.053\end{array}$ & \\
\hline Arch IV & $\begin{array}{l}4.187 \\
0.001 \%\end{array}$ & $\begin{array}{l}2.947 \\
0.016^{*}\end{array}$ & $\begin{array}{l}1.257 \\
0.207\end{array}$ \\
\hline & Arch I & Arch II & Arch III \\
\hline
\end{tabular}

\begin{tabular}{|c|c|c|c|}
\hline Arch II & $\begin{array}{l}1.375 \\
0.179\end{array}$ & & \\
\hline Arch III & $\begin{array}{l}2.507 \\
0.014 \%\end{array}$ & $\begin{array}{l}1.177 \\
0.243\end{array}$ & \\
\hline Arch IV & $\begin{array}{l}3.547 \\
0.004 \%\end{array}$ & $\begin{array}{l}2.279 \\
0.039 *\end{array}$ & $\begin{array}{l}1.198 \\
0.259\end{array}$ \\
\hline & Arch I & Arch II & Arch III \\
\hline
\end{tabular}

\begin{tabular}{|c|c|c|c|}
\hline Arch II & $\begin{array}{l}1.308 \\
0.222\end{array}$ & & \\
\hline Arch III & $\begin{array}{l}2.095 \\
0.126\end{array}$ & $\begin{array}{l}0.816 \\
0.488\end{array}$ & \\
\hline Arch IV & $\begin{array}{l}2.285 \\
0.118\end{array}$ & $\begin{array}{l}1.034 \\
0.405\end{array}$ & $\begin{array}{l}0.276 \\
0.785\end{array}$ \\
\hline & Arch I & Arch II & Arch III \\
\hline
\end{tabular}

Legend: $\mathrm{T}$ values followed by the $\mathrm{P}$ values in italics *: $\mathrm{P}$ value $<0.05$

Table 7: $\left(^{*}\right)$ Khi-2 values, (®) t- value for bootstrap test and P-value comparing prevalence and mean abundance: For E. minitus $(\mathbf{a}, \mathbf{b})$; For E. imbachi $(\mathbf{C}, \mathbf{d})$ and For E. chibami $(\mathbf{e}, \mathbf{f})$ for anterior and posterior hemibranchias ( $\mathrm{AH}$ and $\mathrm{PH}$ ).

a)

\begin{tabular}{|c|c|c|c|c|c|c|c|}
\hline$P H-I$ & $\begin{array}{l}16.877^{\circ} \\
P<0.01\end{array}$ & & & & & & \\
\hline \multirow[t]{2}{*}{$\mathrm{AH}-\mathrm{II}$} & $0.102^{*}$ & $19.048 *$ & & & & & \\
\hline & $P=0.05$ & $P=0.01$ & & & & & \\
\hline \multirow[t]{2}{*}{$\mathrm{PH}-\mathrm{II}$} & $26.813^{*}$ & $1.461 *$ & $29.340^{*}$ & & & & \\
\hline & $P=0.01$ & $P=0.05$ & $\mathrm{P}=0.01$ & & & & \\
\hline \multirow[t]{2}{*}{$\mathrm{AH}-\mathrm{III}$} & $0.332 *$ & $13.071^{*}$ & $0.796^{*}$ & $22.236^{*}$ & & & \\
\hline & $P=0.05$ & $P=0.01$ & $P=0.05$ & $P \propto 0.01$ & & & \\
\hline \multirow[t]{2}{*}{ PHI-III } & $19.946^{*}$ & $0.160^{*}$ & $22.243^{*}$ & $0.657^{*}$ & $15.868^{*}$ & & \\
\hline & $P=0.01$ & $P=0.05$ & $P=0.01$ & $P=0.05$ & $P=0.01$ & & \\
\hline \multirow[t]{2}{*}{$\mathrm{AH}-\mathrm{IV}$} & $1.190^{*}$ & $9.890^{*}$ & $1.961^{*}$ & $18.231^{*}$ & $0.271^{*}$ & $12.398^{*}$ & \\
\hline & $P=0.01$ & $P=0.01$ & $P=0.05$ & $\mathrm{P} \approx 0.01$ & $P>0.05$ & $\mathrm{P} \approx 0.01$ & \\
\hline \multirow[t]{3}{*}{ PH - IV } & $34.766^{*}$ & $4.167^{*}$ & $37.500^{*}$ & $0.713^{*}$ & $29.743^{*}$ & $2.716^{*}$ & $25.253^{*}$ \\
\hline & $P=0.01$ & $P=0.05$ & $\mathrm{P}=0.01$ & $P=0.05$ & $P=0.01$ & $P=0.05$ & $P=0.01$ \\
\hline & AH-I & PH-I & $\mathrm{AH}-\mathrm{II}$ & PH - II & $\mathrm{AH}-\mathrm{III}$ & PH - III & $\mathrm{AH}-\mathrm{IV}$ \\
\hline
\end{tabular}


b)

\begin{tabular}{|c|c|c|c|c|c|c|c|}
\hline \multirow[t]{2}{*}{$P H-I$} & 0.769 용 & & & & & & \\
\hline & $P>0,05$ & & & & & & \\
\hline \multirow{2}{*}{$\mathrm{AH}-\mathrm{II}$} & $0,769 \circledast$ & $3.907 \otimes$ & & & & & \\
\hline & $P=0,05$ & $\mathrm{P}=0,01$ & & & & & \\
\hline \multirow[t]{2}{*}{$\mathrm{PH}-\mathrm{II}$} & $5.507 \AA$ & B 1.586 & $4.599 \mathrm{~B}$ & & & & \\
\hline & $\mathrm{P}=0,01$ & $P>0,05$ & $P=0,01$ & & & & \\
\hline \multirow[t]{2}{*}{ AH - III } & 3.1138 & $3.095 \otimes$ & 2.1908 & $4.648 \otimes$ & & & \\
\hline & $P=0,01$ & $P=0,01$ & $\mathrm{P}=0,05$ & $P=0,01$ & & & \\
\hline \multirow[t]{2}{*}{$\mathrm{PH}-\mathrm{III}$} & $5.663 \otimes$ & B1.586 & $4.753 \otimes$ & 0.4308 & $5.082 \otimes$ & & \\
\hline & $P=0,01$ & $P>0,05$ & $P=0,01$ & $P>0,05$ & $P=0,01$ & & \\
\hline \multirow[t]{2}{*}{$\mathrm{AH}-\mathrm{IV}$} & $3.85 \otimes$ & 2.1048 & $2.928 \&$ & 3.9048 & 1.2348 & $4.447 \circledast$ & \\
\hline & $\mathrm{P} \approx 0,01$ & $P=0,05$ & $P=0,05$ & $\mathrm{P}=0,01$ & $P=0,05$ & $\mathrm{P} \approx 0,01$ & \\
\hline \multirow[t]{3}{*}{ PH - IV } & 5.9618 & 2.0248 & $5.055 \otimes$ & 1.6648 & 5.8518 & 1.4918 & $5.438 \otimes$ \\
\hline & $\mathrm{P}=0,01$ & $P=0,05$ & $\mathrm{P}=0,01$ & $P>0,05$ & $\mathrm{P}=0,01$ & $P>0,05$ & $\mathrm{P}=0,01$ \\
\hline & AH-I & PH-I & $\mathrm{AH}-\mathrm{II}$ & PH - II & AH - III & PH - III & $\mathrm{AH}-\mathrm{IV}$ \\
\hline
\end{tabular}

c)

\begin{tabular}{|c|c|c|c|c|c|c|c|}
\hline$P H-I$ & $\begin{array}{l}15,868^{*} \\
P<0,01\end{array}$ & & & & & & \\
\hline \multirow[t]{2}{*}{$\mathrm{AH}-\mathrm{II}$} & $0.078^{*}$ & $17.825^{*}$ & & & & & \\
\hline & $P=0,05$ & $P=0,01$ & & & & & \\
\hline \multirow[t]{2}{*}{$\mathrm{PH}-\mathrm{II}$} & $24.000^{*}$ & $1.033^{*}$ & $26.272^{*}$ & & & & \\
\hline & $P=0,01$ & $P>0,05$ & $P=0,01$ & & & & \\
\hline \multirow[t]{2}{*}{$\mathrm{AH}-\mathrm{III}$} & $0.071^{*}$ & $14.063^{*}$ & $0.298^{*}$ & $21.869^{*}$ & & & \\
\hline & $P=0,05$ & $P=0,01$ & $P>0,05$ & $P=0,01$ & & & \\
\hline \multirow[t]{2}{*}{$\mathrm{PH}-\mathrm{III}$} & $18.919^{*}$ & $0.162^{*}$ & $21.007^{*}$ & $0.378^{*}$ & $16.978^{*}$ & & \\
\hline & $\mathrm{P}<0,01$ & $P>0,05$ & $\mathrm{P}=0,01$ & $P=0,05$ & $P=0,01$ & & \\
\hline \multirow[t]{2}{*}{$\mathrm{AH}-\mathrm{IV}$} & $0.071^{*}$ & $14.063^{*}$ & $0.298^{*}$ & $21.869 *$ & $0.000^{*}$ & $16.978^{*}$ & \\
\hline & $P=0,05$ & $\mathrm{P}=0,01$ & $P=0,05$ & $P=0,01$ & $P=0,05$ & $\mathrm{P}=0,01$ & \\
\hline \multirow[t]{3}{*}{ PH - IV } & $33.980^{*}$ & $4.340^{*}$ & $36.526^{*}$ & $1.169^{*}$ & $31.562^{*}$ & $2.852^{*}$ & $31.562^{*}$ \\
\hline & $\mathrm{P}=0,01$ & $P=0,05$ & $\mathrm{P}=0,01$ & $P=0,05$ & $\mathrm{P}=0,01$ & $P=0,05$ & $\mathrm{P}=0,01$ \\
\hline & AH-I & PH-I & AH-II & PH - II & AH - III & PH - III & $\mathrm{AH}-\mathrm{IV}$ \\
\hline
\end{tabular}


d)

\begin{tabular}{|c|c|c|c|c|c|c|c|}
\hline$P H-I$ & $\begin{array}{l}4,430 \text { (8) } \\
P<0,01\end{array}$ & & & & & & \\
\hline \multirow[t]{2}{*}{$\mathrm{AH}-\mathrm{II}$} & B1.265ß & B) 3.607 B & & & & & \\
\hline & $P>0,05$ & $P=0,01$ & & & & & \\
\hline \multirow{2}{*}{$\mathrm{PH}-\mathrm{II}$} & \&5.163\& & B 1.1498 & 4.5048 & & & & \\
\hline & $P=0,01$ & $P>0,05$ & $\mathrm{P}=0,01$ & & & & \\
\hline \multirow[t]{2}{*}{ AH - III } & $2.695 \otimes$ & $2.305 \otimes$ & 1.5598 & 3.3728 & & & \\
\hline & $\mathrm{P}=0,01$ & $\mathrm{P}=0,05$ & $P=0,05$ & $\mathrm{P}=0,01$ & & & \\
\hline \multirow[t]{2}{*}{$\mathrm{PH}-\mathrm{III}$} & 5.2878 & 1.2128 & 4.6718 & $-0.055 \otimes$ & $3.56 \otimes 2$ & & \\
\hline & $P=0,01$ & $P=0,05$ & $P=0,01$ & $P=0,05$ & $P=0,01$ & & \\
\hline \multirow[t]{2}{*}{$\mathrm{AH}-\mathrm{IV}$} & $3.621 @$ & $1.388 @$ & 2.6248 & $2.619 \circledast$ & $1.118 \otimes$ & $2.830 \AA$ & \\
\hline & $P=0,01$ & $P=0,05$ & $\mathrm{P}=0,01$ & $\mathrm{P}=0,01$ & $P=0,05$ & $P=0,01$ & \\
\hline \multirow[t]{3}{*}{ PH - IV } & 5.8148 & 2.1448 & $5.345 \otimes$ & 0.8648 & $4.408 \otimes$ & $1.088 \otimes$ & $3.878 \otimes$ \\
\hline & $P=0,01$ & $P=0,05$ & $P=0,01$ & $P=0,05$ & $P=0,01$ & $P=0,05$ & $P \approx 0,01$ \\
\hline & AH-I & PH-I & AH- II & PH-II & AH - III & PH - III & $\mathrm{AH}-\mathrm{IV}$ \\
\hline
\end{tabular}

e)

\begin{tabular}{|c|c|c|c|c|c|c|c|}
\hline$P H-I$ & $\begin{array}{l}14.924^{*} \\
P<0.01\end{array}$ & & & & & & \\
\hline \multirow[t]{2}{*}{$\mathrm{AH}-\mathrm{II}$} & $0.480^{*}$ & $10.390^{*}$ & & & & & \\
\hline & $\mathrm{P}=0.05$ & $P=0.01$ & & & & & \\
\hline \multirow[t]{2}{*}{$\mathrm{PH}-\mathrm{II}$} & $33.653^{*}$ & $4.762^{*}$ & $27.214^{*}$ & & & & \\
\hline & $P=0.01$ & $P=0.05$ & $P=0.01$ & & & & \\
\hline \multirow[t]{2}{*}{ AH - III } & $4.596^{*}$ & $3.241^{*}$ & $2.154^{*}$ & $15.174^{*}$ & & & \\
\hline & $P=0.05$ & $P>0.05$ & $P>0.05$ & $P=0.01$ & & & \\
\hline \multirow[t]{2}{*}{$\mathrm{PH}-\mathrm{III}$} & $44.000^{*}$ & $10.187^{*}$ & $36.946^{*}$ & $1.190^{*}$ & $23.253^{*}$ & & \\
\hline & $P=0.01$ & $P=0.01$ & $P=0.01$ & $P>0.05$ & $P=0.01$ & & \\
\hline \multirow[t]{2}{*}{$\mathrm{AH}-\mathrm{IV}$} & $5.473^{*}$ & $2.564^{*}$ & $2.778^{*}$ & $13.752^{*}$ & $0.041^{*}$ & $21.569^{*}$ & \\
\hline & $P=0.05$ & $P>0.05$ & $P>0.05$ & $P<0.01$ & $P=0.05$ & $P=0.01$ & \\
\hline \multirow[t]{3}{*}{ PH - IV } & $44.000^{*}$ & $10.187^{*}$ & $36.946^{*}$ & $1.190^{*}$ & $23.253^{*}$ & $0.000^{*}$ & $21.569^{*}$ \\
\hline & $P=0.01$ & $P=0.01$ & $P=0.05$ & $P=0.05$ & $\mathrm{P}=0.01$ & $P=0.05$ & $P=0.01$ \\
\hline & AH-I & PH-I & $\mathrm{AH}$ - II & PH - II & AH - III & PH - III & $\mathrm{AH}-\mathrm{IV}$ \\
\hline
\end{tabular}


f)

\begin{tabular}{|c|c|c|c|c|c|c|c|}
\hline$P H-I$ & $\begin{array}{l}2.765 \text { ( } \\
P>0.05\end{array}$ & & & & & & \\
\hline \multirow[t]{2}{*}{$\mathrm{AH}-\mathrm{II}$} & 1.2748 & $1.858 \otimes$ & & & & & \\
\hline & $P>0.05$ & $P=0.05$ & & & & & \\
\hline \multirow[t]{2}{*}{ PH - II } & 3.1148 & $2.665 \otimes$ & $2.382 B$ & & & & \\
\hline & $P=0.05$ & $P=0.05$ & $P=0.05$ & & & & \\
\hline \multirow[t]{2}{*}{$\mathrm{AH}-\mathrm{III}$} & $2.297 \otimes$ & 1.3208 & 1.1868 & 2.5948 & & & \\
\hline & $P=0.05$ & $P=0.05$ & $P=0.05$ & $P>0.05$ & & & \\
\hline \multirow[t]{2}{*}{$\mathrm{PH}-\mathrm{III}$} & 3.1328 & 2.7768 & 2.4098 & 0.2948 & 2.6548 & & \\
\hline & $P>0.05$ & $P=0.01$ & $P>0.05$ & $P>0.05$ & $P>0.05$ & & \\
\hline \multirow[t]{2}{*}{$\mathrm{AH}-\mathrm{IV}$} & 2.3498 & 1.7028 & 1.2478 & $3.586 \mathrm{~B}$ & $0.028 B$ & $3.660 \mathrm{~B}$ & \\
\hline & $P>0.05$ & $P>0.05$ & $P>0.05$ & $P=0.05$ & $P>0.05$ & $P=0.01$ & \\
\hline \multirow[t]{3}{*}{ PH - IV } & $3.162 \otimes$ & 3.1088 & $2.455 \otimes$ & 0.8568 & $2.773 \otimes$ & $0.479 \circledast$ & $3.852 @$ \\
\hline & $P>0.05$ & $P=0.05$ & $P>0.05$ & $P=0.05$ & $P>0.05$ & $P=0.05$ & $P=0.05$ \\
\hline & AH-I & PH-I & $\mathrm{AH}$ - II & $\mathrm{PH}-\mathrm{II}$ & $\mathrm{AH}$ - III & PH - III & $\mathrm{AH}-\mathrm{IV}$ \\
\hline
\end{tabular}

Table 8: $\left.{ }^{*}\right)$ Forbes Index, $(* *)$ Khi-2 and $P$ values comparing prevalence of the combinations of pairs of the three principal parasites species of P. obscura.

\section{a) E. minitus / E. imbachi}

\begin{tabular}{lllll}
\hline & Arch1 & Arch2 & Arc3 & Arch4 \\
\hline Sector 1 & $1,13^{*}$ & $1,38^{*}$ & $1,36^{*}$ & $1,64^{*}$ \\
& $4,22^{* *}$ & $24,59^{* *}$ & $5,19^{* *}$ & $4,56^{* *}$ \\
& $\mathrm{P}<0,05$ & $\mathrm{P}<0,01$ & $\mathrm{P}<0,05$ & $\mathrm{P}<0,05$ \\
\hline Sector 2 & $1,27^{*}$ & $1,24^{*}$ & $1,46^{*}$ & $1,48^{*}$ \\
& $9,61^{* *}$ & $16,14^{* *}$ & $21,16^{* *}$ & $7,41^{* *}$ \\
& $\mathrm{P}<0,01$ & $\mathrm{P}<0,01$ & $\mathrm{P}<0,01$ & $\mathrm{P}<0,01$ \\
\hline Sector 3 & $1,45^{*}$ & $1,45^{*}$ & $1,25^{*}$ & $1,36^{*}$ \\
& $1,51^{* *}$ & $0,82^{* *}$ & $5,22^{* *}$ & $6,90^{* *}$ \\
& $\mathrm{P}>0,05$ & $\mathrm{P}>0,05$ & $\mathrm{P}<0,05$ & $\mathrm{P}<0,05$ \\
\hline Sector 4 & $1,25^{*}$ & $1,47^{*}$ & $1,35^{*}$ & $1,5^{*}$ \\
& $4,83^{* *}$ & $9,90^{* *}$ & $0,85^{* *}$ & $9,70^{* *}$ \\
& $\mathrm{P}<0,05$ & $\mathrm{P}<0,01$ & $\mathrm{P}>0,05$ & $\mathrm{P}<0,01$ \\
\hline Sector 5 & $1,18^{*}$ & $1,3^{*}$ & $1,96^{*}$ & $2,07^{*}$ \\
& $1,93^{* *}$ & $2,90^{* *}$ & $1,80^{* *}$ & $5,26^{* *}$ \\
& $\mathrm{P}>0,05$ & $\mathrm{P}>0,05$ & $\mathrm{P}>0,05$ & $\mathrm{P}<0,05$ \\
\hline
\end{tabular}


b) E. minitus / E. chibami

\begin{tabular}{lllll}
\hline & Arch1 & Arch2 & Arch3 & Arch4 \\
\hline Sector 1 & $1,45^{*}$ & $1,2^{*}$ & $1,57^{*}$ & $1,75^{*}$ \\
& $2,80^{* *}$ & $24,59^{* *}$ & $5,19^{* *}$ & $4,56^{* *}$ \\
& $\mathrm{P}>0,05$ & $\mathrm{P}<0,01$ & $\mathrm{P}<0,05$ & $\mathrm{P}<0,05$ \\
\hline Sector 2 & $1,51^{*}$ & $1,35^{*}$ & $1,36^{*}$ & $1,85^{*}$ \\
& $19,23^{* *}$ & $16,14^{* *}$ & $21,16^{* *}$ & $7,41^{* *}$ \\
& $\mathrm{P}<0,01$ & $\mathrm{P}<0,01$ & $\mathrm{P}<0,01$ & $\mathrm{P}<0,01$ \\
\hline Sector 3 & $1,58^{*}$ & $1,83^{*}$ & $1,43^{*}$ & $1,46^{*}$ \\
& $13,16^{* *}$ & $0,82^{* *}$ & $5,22^{* *}$ & $6,90^{* *}$ \\
& $\mathrm{P}<0,01$ & $\mathrm{P}>0,05$ & $\mathrm{P}<0,05$ & $\mathrm{P}<0,01$ \\
\hline Sector 4 & $1,38^{*}$ & $1,39^{*}$ & $1,15^{*}$ & $0,92^{*}$ \\
& $5,07^{* *}$ & $9,90^{* *}$ & $0,85^{* *}$ & $9,70^{* *}$ \\
& $\mathrm{P}<0,05$ & $\mathrm{P}<0,05$ & $\mathrm{P}>0,05$ & $\mathrm{P}<0,01$ \\
\hline Sector 5 & $1,12^{*}$ & $1,99^{*}$ & $1,87^{*}$ & $1,54^{*}$ \\
& $1,71^{* *}$ & $2,90^{* *}$ & $1,80^{* *}$ & $5,26^{* *}$ \\
& $\mathrm{P}>0,05$ & $\mathrm{P}>0,05$ & $\mathrm{P}>0,05$ & $\mathrm{P}<0,05$ \\
\hline
\end{tabular}

c) E. imbachi / E. chibami

\begin{tabular}{|c|c|c|c|c|}
\hline & Arch1 & Arch2 & Arch3 & Arch4 \\
\hline \multirow[t]{3}{*}{ Sector 1} & $1,21^{*}$ & $1,38^{*}$ & $1,7^{*}$ & $1,48^{*}$ \\
\hline & $2,80^{* *}$ & $5,19^{* *}$ & $3,61 * *$ & $2,49^{* *}$ \\
\hline & $P>0,05$ & $P<0,05$ & $P>0,05$ & $P>0,05$ \\
\hline \multirow[t]{3}{*}{ Sector 2} & $1,41^{*}$ & $1,23^{*}$ & $2,32^{*}$ & $1,31^{*}$ \\
\hline & $19,23^{* *}$ & $7,13^{* *}$ & $4,05 * *$ & $8,46^{* *}$ \\
\hline & $P<0,01$ & $P<0,01$ & $P<0,05$ & $P<0,01$ \\
\hline \multirow[t]{3}{*}{ Sector 3} & $1,34^{*}$ & $1,22^{*}$ & $1,39^{*}$ & $1,33^{*}$ \\
\hline & $13,16^{* *}$ & $8,88^{* *}$ & $4,35^{* *}$ & $5,82^{* *}$ \\
\hline & $P<0,01$ & $P<0,01$ & $P<0,05$ & $P<0,05$ \\
\hline \multirow[t]{3}{*}{ Sector 4} & $1,54^{*}$ & $1,17^{*}$ & $1,55^{*}$ & $1,74^{*}$ \\
\hline & $5,07 * *$ & $1,80^{* *}$ & $0,05^{* *}$ & $0,36 * *$ \\
\hline & $P<0,05$ & $P>0,05$ & $\mathrm{P}>0,05$ & $P>0,05$ \\
\hline \multirow[t]{3}{*}{ Sector 5} & $1,23^{*}$ & $1,42^{*}$ & $1,46^{*}$ & $0,77^{*}$ \\
\hline & $1,71^{* *}$ & $5,36^{* *}$ & $0,83^{* *}$ & $0,05^{* *}$ \\
\hline & $P>0,05$ & $P<0,05$ & $\mathrm{P}>0,05$ & $P>0,05$ \\
\hline
\end{tabular}


Table 9: (*) Forbes index, (**) Khi-2 and $P$ values comparing pairs parasites prevalences per gill arch zones: a) E. minitus vs $E$. imbachi; b) $E$. minitus vs $E$. chibami ; c) E. imbachi vs $E$. chibami.

a.

\begin{tabular}{llll}
\hline & Distal zone & Median zone & Basal zone \\
\hline Arch1 & $1,290^{8}$ & $1,154^{8}$ & $1,154^{8}$ \\
& $11,20^{* 8}$ & $11,31^{8 *}$ & $12,18^{* 8}$ \\
& $\mathrm{P}<0,001$ & $\mathrm{P}<0,001$ & $\mathrm{P}<0,001$ \\
\hline Arch2 & $1,515^{8}$ & $1,184^{8}$ & $1,173^{8}$ \\
& $15,84^{8 *}$ & $20,46^{* 8}$ & $7,12^{* 8}$ \\
& $\mathrm{P}<0,0001$ & $\mathrm{P}<0,001$ & $\mathrm{P}<0,01$ \\
\hline Arch3 & $1,319^{8}$ & $0,952^{8}$ & $1,257^{8}$ \\
& $10,74^{8 *}$ & $27,73^{* 8}$ & $10,74^{8 *}$ \\
& $\mathrm{P}<0,001$ & $\mathrm{P}<0,01$ & $\mathrm{P}<0,01$ \\
\hline Arch4 & $1,466^{8}$ & $1,429^{8}$ & $1,667^{*}$ \\
& $13,72^{8 *}$ & $4,39^{* 8}$ & $9,82^{* 8}$ \\
& $\mathrm{P}<0,001$ & $\mathrm{P}<0,03$ & $\mathrm{P}<0,01$ \\
\hline
\end{tabular}

b.

\begin{tabular}{|c|c|c|c|}
\hline & Distal zone & Median zone & Basal zone \\
\hline \multirow[t]{3}{*}{ Archl } & $1,429^{8}$ & $1,351^{8}$ & $1,316^{8}$ \\
\hline & 10,7488 & 21,3388 & $11,52^{88}$ \\
\hline & $\mathrm{P}<0,001$ & $\mathrm{P}<0,001$ & $\mathrm{P}<0,001$ \\
\hline \multirow{3}{*}{ Arch2 } & $1,601^{8}$ & $1,267^{8}$ & $1,389^{8}$ \\
\hline & $3,54^{88}$ & $8,32 * 8$ & 4,9988 \\
\hline & $P=0,5$ & $\mathrm{P}=0,01$ & $\mathrm{P}<0,05$ \\
\hline \multirow[t]{3}{*}{ Arch3 } & $1,444^{8}$ & $2,554^{8}$ & $1,478^{\circ}$ \\
\hline & 588 & $2,908 \%$ & $2,14 \% 8$ \\
\hline & $P<0,05$ & $P=0,05$ & $P=0,05$ \\
\hline \multirow[t]{3}{*}{ Arch4 } & $2,632^{8}$ & $1,287^{8}$ & $3,247^{8}$ \\
\hline & $9,49 * 8$ & $5,93 * 8$ & $2,48 * 8$ \\
\hline & $\mathrm{P} \Leftarrow 0,01$ & $P=0.05$ & $P>0.05$ \\
\hline
\end{tabular}

c.

\begin{tabular}{|c|c|c|c|}
\hline & Distal zone & Median zone & Basal zone \\
\hline \multirow{3}{*}{ Archl } & $1,613^{8}$ & $1,154^{8}$ & $1,319^{*}$ \\
\hline & $7,99 * 8$ & $9,79 * 8$ & $9,08 * 8$ \\
\hline & $P=0,05$ & $\mathrm{P}<0,01$ & $\mathrm{P}<0,01$ \\
\hline \multirow{3}{*}{ Arch2 } & $1,122^{8}$ & $1,150^{8}$ & $1,054^{8}$ \\
\hline & $0,25 * 8$ & 3,6588 & $0,08 * 8$ \\
\hline & $P=0,05$ & $P>0,05$ & $P=0,05$ \\
\hline \multirow[t]{3}{*}{ Arch3 } & $1,667^{8}$ & $1,223^{8}$ & $1,282^{8}$ \\
\hline & $8,56 * 8$ & $2,31^{88}$ & $0,10 \% 8$ \\
\hline & $P=0,005$ & $P<0,05$ & $P=0,05$ \\
\hline \multirow[t]{3}{*}{ Arch4 } & $1,989^{8}$ & $1,195^{\circ}$ & $1,573^{8}$ \\
\hline & 3,5588 & $0,63 \approx 8$ & 4,4988 \\
\hline & $P>0,05$ & $P>0,05$ & $P>0,05$ \\
\hline
\end{tabular}




\section{DISCUSSION}

Five species: E. magnus, E. minutus, $E$. imbachi, E. chibami and E. malleus were recorded on the gill filaments of $P$. obscura caught in Lake Ossa, Sanaga Basin. This monogenean richness is similar to the finding of Bilong Bilong et al. (1994) in the Nyong Basin; it confirms the host interchanges between Cameroonian river systems (Thys van den Audenaerde, 1966) which favor not only parasites' transfers (Nack et al., 2015) but also their geographical dispersal.

This parasitism by five congeneric species raises the question of arch partitioning; it also suggests that sympatric speciation is more common than thought and that competition is not the main community structuring force, for it allows similar ecological species to coexist (Lim, 1990). Fish polyparasitism by monogeneans is quite well documented (Boungou et al., 2008; Ibrahim, 2012; Tombi et al., 2014). It is related to many parameters such as sampling effort (Walter et al., 1995), host and parasite phylogenies (Zharikova, 2000), ecology (Zharikova, 2000), water quality (Galli et al., 2001) and vacant niches due to low parasites load (Nack et al., 2018). In this work $E$. magnus and E. malleus with prevalence of $24 \%$ and $58 \%$ respectively had very low mean intensities $(<10)$; they are therefore considered satellite species. Eutrianchoratus chibami and E. imbachi were common (Prev $\geq$ $90 \%)$ but their mean intensities were low $(<$ $35)$; they are considered intermediate. The common E. minutus (Prev = 96\%), although having a low mean intensity (48.9), is considered the core species in this xenocommunity. Therefore, in the Lake Ossa environment, though their prevalence was somewhat high, all these monogenean species mean intensities were very low or low. This fact suggests the absence of competition among these parasites due to their small size when compared with the gill area of the host (Paperna, 1982) and the prevailing low intensity of infection (Tombi and Bilong Bilong, 2004; Nack et al., 2010). Similarly, Šimková and Rohde (2013) stated since vacant niches are available, interspecific competition is unimportant for freshwater and marine gill parasites of fishes. Parachanna obscura individuals captured in Lake Ossa appeared in good condition since their condition factor $\mathrm{K}$ was averagely 1.40 (Froese, 2006); this proves that in this natural environment, parasite specific loads were below dangerous thresholds. In fact, under normal circumstances, although monopisthocotyleans do cause epidermal lesions on their fish hosts, the resulting damage is negligible since fish possess high regenerative abilities (Kinkelin et al., 1985; Kabata, 1985; Cheng, 1986). Paperna (1982) summarized that in fish species with a fast growth [case of P. obscura (Teugels, 2007)], the tissue response makes rapid parasite spreading difficult, and that, from a length of 35 to $38 \mathrm{~mm}$, the gill filaments may harbor high parasite load because the fish acquire good immunity to eliminate them. This statement also relativizes the impact of some high parasite loads observed in this work at the component community level, although the seasonal approach of the intensity still remains unstudied.

Males and females $P$. obscura were similarly infected (prevalence and mean intensity) probably because both sexes live in the same environment (Welcomme and Merona, 1988) and are equally exposed and susceptible to oncomiracidia. Although there was no statistical difference in the infection among host length classes, our findings on $E$. minutus suggests that big/large or old fish accumulate monogeneans over time and consequently represent the parasitic source or reservoir for young individuals (Cable et al., 2002; Blahoua et al., 2009; Ibrahim, 2012; Tombi et al., 2014).

For the right and left body sides, it is suggested that the bilateral symmetric body of this fish and its parasite species support the evenness of the parasite loads of both sides (Soylu et al., 2013). Rohde (1993) related one side fish preference by parasites to the body asymmetry of the host. The symmetric distribution of monogenean gill parasites is a well-documented phenomenon (Nack et al., 2010; Tombi et al., 2010; Soylu et al., 2013), 
while asymmetric distribution is scarce (Hendrix, 1990).

The mean number of the core $(E$. minutus) and the intermediate (E. imbachi) species decreased antero-posteriorly from arch I to arch IV, while for the second intermediate species (E. chibami), this parasite load was the same from arch I to arch IV. Concerning the variation of the mean number of the core and the intermediate species, our results are similar to those of Tombi et al. (2014) for monogenean gill parasites of Oreochromis niloticus, Jerônimo et al. (2013) for monogenean gill parasites of the hybrid patinga, on the first three gill arches when compared with gill arch IV, and Starruscu Bedivan et al. (2008) who noticed that the gill arch I of Barbus meridionalis was more infected by Paradiplozoon tisae. In many other cases, monogenean parasites prefer the two median arches II and III (Tombi et al., 2014). Conversely in Cameroon, Nack et al. (2010) found that Quadriacanthus sp. and Birgiellus kellensis, gill monogeneans of the catfish Clarias camerunensis, prefer arch IV rather than arch I. Many hypotheses have been proposed to explain the gill arch preference by monogeneans on teleosts. Authors like Lo and Morand (2001) argued that the median arches are more infected because of the large volume of water flowing through them, while Buchmann (1989) and Koskivaara and Valtonen (1991) associated this preference to the large surface of colonization offered to parasites. Lo and Morand (2001) previously explained this phenomenon by parasite load which induces the competition and thus the gill selection. Koskivaara and Valtonen (1992) and Guttiérrez and Martorelli (1994) also suggested that the host immunity can influence the microhabitat selection. In the present work, all parasite species had broader niches in the presence of congeners in the infracommunities. Therefore, the different water volume of the ventilating current over the arches, the parasite loads and the large two median arches in the bucco-pharyngeal cavity do not explain the distribution of Eutrianchoratus spp. on the gill arches of $P$. obscura. Dactylogyrid larvae swim actively and then attach to the skin or gills of susceptible fish (Nack et al., 2010; Tombi et al., 2010); the distribution pattern of at least $E$. minutus, the core species of this xenocommunity, suggests that its oncomiracidia are transported by the ventilating water current, that the filaments of the different gill arches exert an anteroposterior sifting effect on the infecting larvae and that posterior arches and hemibranches are less exposed to oncomiracidia than anterior ones. Our results raise the question of whether there are other models of ventilation of gill filaments in teleosteans than that described by Paling (1968).

The study of parasitism prevalence and abundances in function of hemibranch revealed that Eutrianchoratus minitus, E. imbachi and E. chibami prefer anterior hemibranches. Turgut et al. (2006) showed that Dactylogyrus species have a preference for the most ventilated hemibranches. Tombi et al. (2010) showed that Dactylogyrus insolitus preferentially binds to the posterior hemibranches of the arc II of Barbus martorelli whereas D. bopeleti, D. maillardi and $D$. simplex have no preference for hemibranches.

The co-occurrence of the different pairs of the three main monogenean species (E. minitus, E. imbachi, E. chibami) at the sectoral and zonal level showed in most cases random colonization. In many others studies, analyzes of interspecific coexistence have shown no interactions (Morand and Simkova, 2005; Tombi et al., 2011). However, some others studies have revealed positive interactions (Silan and Maillard, 1990; Kooskivaara, 1992). Moreover, Rohde (1994) stated that positive interactions between fish ectoparasites are more frequent than negative ones, although Holmes and Price (1986) argued that negative interactions should be frequent. The lack of interspecific competition in this study is also due to the low parasitic intensities inducing niches vacancy within the gill biotope. Indeed, Buchmann (1989), Koskivaara and Valtonen (1991) argue that a multi-species infestation cannot be accompanied by competition as long as there 
is sufficient space and resources. Price (1980) also considered that as long as there are vacant niches for parasites, interspecific interactions will be rare.

Our finding supports the statement by Koskivaara and Valtonen (1992) that multispecies infection might exist without competition between monogenean species. In our example, there are still enough resources and space in the infracommunities to be exploited in the long term.

\section{Conclusion}

The study allowed to identify 5 species of monogenean gill parasites of $P$. obscura. The analysis of their spatial distribution indicated that there was no sex effect, nor size, neither gill side in the distribution of these parasites. In most cases, there was no significant difference in the parasitism of the different gill arches. But there was an anteroposterior model of accumulation of monogeneans on the arches. No evidence of competition between species of Eutrianchoratus on the gill tissue was found.

\section{COMPETING INTERESTS}

The authors declare that they have no competing interests.

\section{AUTHORS' CONTRIBUTIONS}

JN initiated the project. MY collected fish specimens. JN and MY dissected the fish and mounted the parasites. CFBB, FDMM and JN did the statistical analysis. CFBB and $\mathrm{JN}$ wrote the article. All the authors corrected and approved the final manuscript.

\section{ACKNOWLEDGEMENTS}

The authors thank $\mathrm{Dr}$ Antoine PARISELLE of IRD for his technical assistance and Dr Arnold Roger BITJA NYOM of the Institute of Fisheries and Aquatic Sciences of the University of Douala who identified the host species of the studied monogeneans

\section{REFERENCES}

Bahanak D, Nack J, Pariselle A, Bilong Bilong CF. 2016. Description of three new species of Quadriacanthus (Monogenea: Ancyrocephalidae) gill parasites of Clarias submarginatus (Siluriformes: Clariidae) from Lake Ossa (Littoral region, Cameroon). Zoologia, 33(4): 1-8. DOI 10.1590/S1984 - 4689 Zool 20160044.

Bilong Bilong CF. 1986. Helminthes parasites du Cichlidae Hemichromis fasciatus des eaux douces du Cameroun : Taxonomie, Biologie, Ecologie. Thèse de Doctorat de $3^{\text {e }}$ cycle de l'Université de Yaoundé I. $p$ 213.

Bilong Bilong CF. 1995. Les Monogènes parasites de poissons d'eau douce $d u$ Cameroun : biodiversité et spécificité, biologie des populations inféodées à Hemichromis fasciatus. Thèse de Doctorat d'Etat de l'Université de Yaoundé I. p 341.

Bilong Bilong CF, Birgi E, Euzet L. 1994. Urogyrus cichlidarum gen. nov. sp. nov. Urogyridae fam. nov. monogène parasite de la vessie urinaire de Poissons Cichlidae au Cameroun. Can. J. Zool., 72: 561-566. DOI: 10.1139 /z94-076.

Bilong Bilong CF, Njiné T. 1998. Dynamique de population de trois monogènes parasites d'Hemicromis fasciatus Peters, 1858 dans le Lac Municipal de Yaoundé, et intérêt possible en pisciculture intense. Ann. Fac. Sci. Univ. Yaoundé I, Sér. Sci. Nat. Vie., 34(2): 295-303.

Blahoua KG, N'Douba V, Tidani KK, N'Guessan KJ. 2009. Variations saisonnières des indices épidémiologiques de trois monogènes parasites de Sarotherodon melanotheron (Pisces, Cichlidae) dans le lac Ayamé (Côte d'Ivoire). Sci. Nat., 6(1): 39-47. DOI: $10.4314 /$ scinat.v6i1.48578. 
Boungou M, Kabre GB, Marques A. Sawadogo L. 2008. Dynamics of population of Five Parasitic Monogeneans of Oreochromis niloticus Linné, 1757 in the Dam of Loumbila and possible Interest in intensive pisciculture. Pak. J. Biol. Sci., 11(10): 1317-1323. DOI: $10.3923 /$ pjbs.2017.539.542.

Buchmann K. 1989. Microhabitat of monogenean gill parasites on European eel (Anguilla anguilla). Fol. Parasitol., 36: 321-329.

Bush AO, Lafferty KD, Lotz JM, Shostak AW. 1997. Parasitology meets ecology on its own terms. J. Parasitol., 83: 575583. DOI: $10.2307 / 3284227$.

Cable J, Tinsley RC, Harris PD. 2002. Survival and embryo development of Gyrodactylus gasterostei (Monogenea: Gyrodactylidae). Parasitol., 124: 53-68. DOI: $10.1017 /$ S0031182001008861P.

Cheng CT. 1986. Monogenea: the Monogenetic Trematodes. In General Parasitology ( $2^{\text {nd }}$ edn). Academic Press College Division, Harcourt Brace Jovanovich, Publishers; 273-298.

Combes C. 1995. Interactions Durables. Ecologie et Evolution du Parasitisme. Masson, (Collection écologie, $\left.\mathrm{n}^{\circ} 26\right)$ : Paris, France ; 524.

Dossou C, Euzet L. 1984. Les Monogènes du genre Eutrianchoratus Paperna, 1969 parasites branchiaux d'Ophiocephalus obscurus au Bénin (Afrique). Bull. Soc. Zool. France, 109: 239-247.

Froese R. 2006. Cube law, condition factor and weight-length relationships: history, meta-analysis and recommendations $J$. Appl. Ichthyol., 22: 241-253. DOI: 10.1111/j.1439-0426.2006.00805.x

Galli P, Crosa G, Manniello L, Ortis M, d'Annelio S. 2001. Water quality as a determinant of the composition of fish parasite communities. Hydrobiol., 452:
173-179. DOI: $10.1023 / \mathrm{A}$ : 1011958422446

Guttierrez PA, Martorelli SR. 1994. Seasonality, distribution and preference sites of Demidespermus valenciennesi Guttiérez et Surriano, 1992 (Monogenea: Ancyrocephalidae) in catfish. Res. Rev. Parasitol., 54(4): 259-261. DOI: 10; 1051/Parasite/1985605571.

Hendrix SS. 1990. Attachment and microhabitat of Bothritrema bothi (Monogenea). ICOPA VII, Paris, S6 A. Dynamique des populations parasites. Résumé de la communication.1P.

Holmes JC, Price PW. 1986. Communities of parasites. In Community Ecology: Pattern and Process, Anderson DJ, Kikkawa J (eds). Blackwell Scientific Publications: Victoria; 187-213.

Ibrahim MM. 2012. Variation in Parasitic intracommunity of Tilapia zillii in relation to some biotic and abiotic factors. Int. J. Zool. Res., 8(2): 58-70. DOI: 10.3923/ijzr.2012.59.70.

Jerônimo GT, Gonçalves ELT, Bampi D, Paseto A, de Pâdua SB, Ishikawa MM, Martins ML. 2013. Microhabitat of Lernaea cyprinacea, on the gills of four Brazilian freshwater fish. Neotrop. Helmint., 7(1): 65-74. DOI: 10.1590/51984-29612013005000021

Kabata Z. 1985. Parasites and diseases of fish cultured in the tropics. Taylor and Francis Editions; London and Philadelphia. p 318.

Kinkelin P, de Michel C, Ghittino P. 1985. Précis de Pathologie des Poissons. INRA-OIE: Paris; 348.

Koskivaara M. 1992. Environmental factors affecting monogeneans parasitic on freshwater fishes. Parasitol. Today, 8: 339-342. DOI: $\quad 10.1016 / 0169-$ 4758(92)90069-E.

Koskivaara M, Valtonen ET. 1991. Paradiplozoon homoion (Monogenea) 
and some other gill parasites on roach Rutilus rutilus in central Finland. Aqua. Fennica., 21(2): 137-143. DOI: 10.1007/BF01830435.

Koskivaara M, Valtonen ET. 1992. Dactylogyrus (Monogenea) communities on the gills parasites of roach in three lakes in central Finland. Parasitol., 104: 263-272.

DOI:

org/10.1017/S0031182000061709.

Kpogue DNS, Mensah GA, Fiogbe EM. 2013. A review of biology, Ecology and prospect for Aquaculture of Parachanna obscura. Rev Fish Biol Fisheries, 23: 4150. DOI: 10.1007/S11160-012-9281-7.

Lekeufack Folefack GB, Fomena A. 2013. Structure et dynamique des infracommunautés de Myxosporidies parasites de Ctenopoma petherici Günther, 1864 (Anabantidae), Clarias pachynema Boulenger, 1903 (Clariidae) et Hepsetus odoe (BLOCH, 1794) (Hepsetidae) dans la rivière Sangé au Cameroun. Int. J. Biol. Chem. Sci., 7(6): 2301-2316.

DOI: http://dx.doi.org/10.4314/ijbcs.v7i6.11

Lim LHS 1990. Freshwater Monogeneans of Peninsular Malaysia. Asian Fisheries Sci, 3: 275-285.

Lo CM, Morand S. 2001. Gills parasites of Cephalopholis argus (Teleostei: Serrainidae) from Moorea (French Polynesia): site selection and coexistence. Fol. Parasitol., 48: 30-36. DOI: $10.14411 /$ fp. 2017.020

Morand S., Šimkovà A. 2005. Metapopulation biology of marine fish parasites, in: Marine Parasite. Rohde K. (ed.), CSIRO Publishing, Collingwood, Australia. 302309.

Mukabo Okito G, Cikwanine Kasigwa D, Micha JC, Nshombo Muderhwa Vet Rwakana O, Ribakare Bizuru Nzibonera P, Muhirwa BG, Mboboci Suku DE, Mudimbe Kaluba P. 2017. Ecologie alimentaire de Labeobarbus altianalis (Boulenger, 1900) du Bassin de la rivière Luhoho, en territoire de Kalehe (SudKivu, R.D. Congo). Int. J. Biol. Chem. Sci., 11(1): 208-227. DOI: http://dx.doi.org/10.4314/ijbcs.v11i1.20

Nack J, Bitja Nyom AR, Pariselle A, Bilong Bilong CF. 2015. New evidence of a lateral transfer of monogenean parasite between distant fish hosts in Lake Ossa, South Cameroon: the case of Quadriacanthus euzeti n. sp. $J$. Helminthol., 90: 455-459. DOI: 10.1017/S0022149X15000577.

Nack J, Massende J, Messu Mandeng DF. 2018. Distribution spatiale de deux monogènes du genre Protoancylodiscoides Paperna, 1969 (Dactylogyridea, Ancyrocephalidae) parasites branchiaux de Chrysichthys auratus (Geoffroy Saint-Hilaire, 1808) (Siluriformes, Claroteidae) dans le Lac Ossa (Littoral -Cameroun). J. Appl. Biosci., 121: 12157-12167. DOI: doi.org/10.4314/jab.v121i1.6.

Nack J, Tombi J, Bitja Nyom AR, Bilong Bilong CF. 2010. Sites de fixation de deux monogènes Dactylogyridea parasites branchiaux de Clarias camerunensis: évidence sur le mode d'infestation par les Monopisthocotylea. J. Appli. Biosci., 33: 2076-2083. DOI: 10.4314/jab.v13121.1

Odo GE, Onoja SU, Onyishi GC. 2012. The biology of Parachanna obscura (Osteichthyes: Channidae) in Anambra River, Nigeria. Int. J. Fish. Aquac., 4(8): 154-169. DOI: 10.5897/IJFA11.022.

Olasunkanmi JB and Ipinmoroti MO. 2014.Food of the African snake head (Parachanna obscura) in a protected area. Int. J. Dev. Sustain., 3(1): 177-183. DOI: IJDS13060702.

Paling JE. 1968. A method of estimating the relative volumes of water flowing over 
the different gills of a freshwater fish. $J$. Exp. Biol., 48: 533-544. DOI: 10.1242/jeb.167031

Paperna I. 1969. Monogenetic trematodes of the fish of the Volta Basin and South Ghana. Bull IFAN, Sér A, XXXI: 840880.

Paperna I. 1982. Parasites, infection et maladies du poisson Afrique. CPCA Document technique $\mathrm{N}^{\circ} 7$ : p 202.

Poulin R. 1998. Evolutionary Ecology of Parasites: from Individuals to Communities $\left(1^{\text {st }}\right.$ edn). Chapman \& Hall: 2-6 Boundary Row, London SE1 8HN, UK; 212.

Price PW. 1980. Evolutionary Biology of Parasites. Princeton University Press: Princeton; 265.

Reiczigel J, Zacharias I, Rôzsa L. 2005. A bootstrap test of stochastic quality of two populations. Amer. Statist., 59: 156-161. DOI: 10.1198/000313005X23526.

Rohde K. 1977. A non-competitive mechanism responsible for restricting niches. Zool. Anzeig. 199(3/4): 164-172.

Rohde K. 1993. Ecology of Marine Parasites. An Introduction to Marine Parasitology ( $2^{\mathrm{e}}$ Edn). Cab International: Wallingford. Oxon; p 298.

Rohde K. 1994. Niche restriction in parasites: proximate and ulti-mate causes. Parasitol., 109: 69-84. DOI: org/10.1017/S0031182000085097.

Rôzsa L, Reiczigel J, Majoros G. 2000. Quantifying parasites in samples of hosts. J. Parasitol., 86(2): 228-232. DOI: 10.1645/0022-3395(2000)086[0228: QPISOH]2.0.CO;2.

Silan P, Maillard C. 1990. Comparative structures and dynamics of some populations of helminths parasites of fishes: the sea bass - Diplectanum model. Acta Oecol., 11: 857-874.

Šimková A, Rohde K. (2013). Community stability and instability in ectoparasites of marine and freshwater fish. Rohde, $\mathrm{K}$. ed. The Balance of Nature and Human Impact. Cambridge University Press: Cambridge; 75-87.

Soylu E, Çolak SO, Erdogan F, Erdogan M, Tektus N. 2013. Microhabitat distribution of Pseudodactylogyrus anguillae (Monogenea) Ergasilus gibbus and Ergasilus lizae (Copepoda) on the gills European Eel (Anguilla anguilla). Acta Zool. Bulg., 65(2): 251-257.

Stavrescu-Bedivan MM, Aioanei FT. 2004. Aspects of branchial parasitism in Barbus meridionalis Petenyi Heckel, 1847. (Teleostei: Cyprinidae). Bull. Vet. Med., 65(2): 57-90.

Stiassny MLJ, Teugels GG, Hopkins CD. 2007. Poissons d'Eaux douces et Saumâtres de Basse Guinée, Ouest de l'Afrique Centrale (Volume I). IRD, MnHn, MRAC : Paris ; 800 p.

Teugels GG. 2007. Channidae. In Poissons d'eaux douces et saumâtres de la basse Guinée, ouest de l'Afrique Centrale (Vol. 2), Stiassny LJM, Teugels GG, Hopkins DG (éds). Institut de Recherche pour le Développement (IRD) (Paris, France), Musée National d'Histoire Naturelle (MNHN) (paris, France) \& Musée Royal de l'Afrique Centrale (MRAC) (Tervuren, Belgique); 241246.

Thys van den Audenaerde DFE. 1966. Les Tilapia (Pisces, Cichlidae) du SudCameroun et $\mathrm{du}$ Gabon. Etude systématique. Ann. MRAC, sér In- $8^{\circ}$ Zool. 153: 1- 98.

Tombi J, Bilong Bilong C F, Morand S. 2011. Gill ectoparasites of Barbus martorelli (Teleostean: Cyprinidae) from a tropical watercourse (Cameroon; Africa): conflict or coexistence? Parasite 18: 71 - 78. DOI: 10.1051/parasite/2011181071.

Tombi J, Akoumba JF, Bilong Bilong CF. 2014. The monogenean community on 
the gills of Oreochromis niloticus from Melen fish Station in Yaoundé, Cameroon. Int. J. Mod. Biol. Res., 2: 1623.

Tombi J, Bilong Bilong CF. 2004. Distribution of gill parasites of the freshwater fish Barbus martorelli Roman, 1971 (Teleostei: Cyprinidae) and tendency to inverse intensity evolution between Myxosporidia and Monogenea as a function of the host age. Rev. Elev. Méd. Vét. Pays. Trop., 57(12): 71-76. DOI: 10. 19182/remvt.31.523.

Tombi J, Nack J, Bilong Bilong CF. 2010. Spatial distribution of Monogenean and Myxosporidian gill parasites of Barbus martorelli Roman, 1971 (Teleostei: Cyprinid): The role of intrinsic factors. Afr. J. Agric. Res., 5(13): 1662-1669. DOI: 10.5897/AJAR09.093.

Turgut E, Shinn A, Wootten R. 2006. Spatial distribution of Dactylogyrus (Monogenean) on the gills of the host fish. Turk. J. Fish. Aquat. Sci., 6: 93-98. DOI: 10.1111/j.1365-. 2109.2009.02235.x.

Valtonen ET, Holmes JC, Koskivaara M. 1997. Eutrophication, pollution and fragmentation: effects on parasite communities in roach (Rutilus rutilus) and perch (Perca fluviatilis) in four lakes in central Finland. Can. J. Fish. Aquat. Sci., 54: 572-585. DOI: org/10.1139/f96306.
Vodounnou DSJV, Kpogue DNS. Akpo Y, Lagnika M. Fiogbe ED. 2017. Determination of sexual dimorphism of African snakehead (Parachanna obscura): morphometric and meristic parameters, weight-length relationship and condition factor. Int. J. Biol. Chem. Sci., 11(4): 1742-1752. DOI : http://dx.doi.org/10.4314/ijbcs.v11i4.26

Walter BA, Clayton DH, Cotgreave PC, Gregory RD, Price RD. 1995. Sampling effort and parasites species richness. Parasitol. Today, 11: 306-310. DOI: org/10.1016/0169-4758 (95)80047-6.

Welcomme RL, de Merona B. 1988. Fish communities of rivers. In Biology and Ecology of African Freshwater Fishes, Levêque C, Bruton MN, Ssentongo GW (eds). ORSTOM, Travaux et Documents 216: Paris; 251-276.

Whenu OO, Fagade SO. 2012. Dietary pattern of the population of Parachanna obscura (Gunther, 1861) in River Owo, south west, Nigeria. J Fish Aquat Sci., 7(1): 49-56.

DOI: $10.3923 /$ jfas.2012.49.56

Zango P, Tomedi MTE, Efole TE, Tiogue CT, Nguenga D, Kamanke Kamanke SM, Mikolasek O, Tchoumboue J. 2016. Performances de reproduction du poisson chat endogène du Cameroun Clarias jaensis (Boulenger, 1909) en milieu contrôlé. Int. J. Biol. Chem. Sci., 10(2): 533-542. DOI : http://dx.doi.org/10.4314/ijbcs.v10i2.7 